\title{
Drag on two coaxial, nonuniformly structured flocs in a uniform flow field
}

\author{
Jyh-Ping Hsu *, Shu-Jen Yeh, Duu-Jong Lee \\ Department of Chemical Engineering, National Taiwan University, Taipei 10617, Taiwan
}

Received 15 March 2005; accepted 21 May 2005

Available online 15 July 2005

\begin{abstract}
The drag on two coaxial flocs of nonuniform structure in a Newtonian fluid is evaluated for Reynolds number ranges from 0.1 to 40. A two-layer model is adopted to simulate various possible structures of a floc. The influences of the key parameters of the problem under consideration, including the separation distance between two flocs, the Reynolds number, and the ratios (inner radius/outer radius) and (permeability of outer layer/permeability of inner layer), on the drag acting on two flocs are investigated. We show that if Reynolds number is small, the drag on the leading floc is about the same as that on the rear floc. However, if Reynolds number is sufficiently large, because wakes are formed in the rear region of the leading floc, the drag on it is greater than that on the rear floc. For a fixed mean permeability, the more nonuniform the floc structure is, the more important is its influence on the drag, and the more appreciable the deviation of the drag coefficient-Reynolds number curve from a Stokes'-law-like relation.
\end{abstract}

(C) 2005 Elsevier Inc. All rights reserved.

Keywords: Drag; Two coaxial flocs; Nonuniform structure; Two-layer model

\section{Introduction}

The linear size of the floc formed in a typical water and wastewater treatment process is on the order of 100 to $1000 \mu \mathrm{m}$. Because the Reynolds number for the sedimentation of such an entity is considerably larger than that required for creeping flow, Stokes' law is usually inapplicable [1]. According to Neale et al. [2], the drag on a porous spherical particle moving with a constant velocity $\mathbf{V}$ in an infinite, stagnant Newtonian fluid can be expressed as

$F=\left(\frac{1}{2} \rho V^{2}\right)\left(\pi r_{1}^{2}\right) C_{\mathrm{D}} \Omega$,

where $C_{\mathrm{D}}$ is the drag coefficient, $r_{1}$ the radius of the particle, $\rho$ is fluid density, and $0 \leqslant \Omega \leqslant 1$ is a correction factor taking the porous structure of the particle into account. $C_{\mathrm{D}}$ is a function of Reynolds number $R e$ and the sphericity of the floc [3-5]. For a rigid sphere of radius $r_{1}$ under creeping flow condition, to which Stokes' law is applicable, the drag acting

\footnotetext{
* Corresponding author. Fax: +886 223623040

E-mail address: jphsu@ntu.edu.tw (J.-P. Hsu).
}

on it, $F_{\mathrm{S}}$, can be expressed as $F_{\mathrm{S}}=6 \pi \mu r_{1} V$, and we have $C_{\mathrm{D}}=24 / R e$ and $\Omega=1$, where $\mu$ is the viscosity of fluid. Apparently, for the same size, the drag on a porous sphere is smaller than that on a rigid sphere. Neale et al. [2] were able to derive an analytical expression for $\Omega$ as a function of the diameter and the permeability of the floc for a uniformly structured floc under creeping flow condition. The result derived was justified experimentally by Matsumoto and Suganuma [6].

Because floc formation involves nonlinear, stochastic processes its structure is of a complicated nature. Assuming a uniform, porous structure, Wu and Lee [7-11] evaluated the drag on a spherical floc. Other types of structure models were also available in the literature. Jorand et al. [12], for example, proposed a three-layered floc model, and a multilayer model was proposed by Veerapanenim and Wiesner. $\mathrm{Li}$ and Ganczarczyk observed that, depending upon the contents and the formation mechanism, floc could have either a less or more permeable outer layer [13,14]. Based on their observation, Hsu and Hsieh used a two-layer model to simulate various possible nonuniform floc structures, and the sedimentation of floc for following geometries were dis- 
cussed: a spherical [15] and a spheroid [16] floc in an infinite Newtonian fluid, a spherical floc along the axis of a cylindrical tube filled with a Newtonian fluid [17], and a spherical floc toward a plane [18]. Adopting a free surface cell model, $\mathrm{Li}$ [19] estimated the drag on a two-layered floc in a floc dispersion; the effects of floc concentration on the flow field and on the drag were examined. Stimson and Jeffery [20] concluded that under creeping flow conditions, the drag on two rigid, coaxial spheres in an infinite viscous fluid are the same, and can be expressed as

$F_{\mathrm{S}}=3 \pi \mu d_{\mathrm{r}} V \gamma$

where $\gamma$ denotes the ratio between the drag exerted on either sphere and the Stokes' law. In an attempt to measure the drag on two interacting rigid particles, Rowe and Henwood [21], Lee [22], and Tsuji et al. [23] applied a pendulum method and water channel flow; the Reynolds number ranged from 500 to 10,000 . Due to the fluctuating nature of the data gathered, however, only a general trend between drag coefficient and the separation distance between two particles could be obtained. Zhu et al. [24] developed a microforce measuring system, which could be used directly to measure the interaction force between two rigid particles for Reynolds number ranges from 20 to 130 . Their method was adopted by Liang et al. [25] and Chen and $\mathrm{Wu}$ [26] to measure the drag on two rigid spheres.

In this study the drag on two coaxial flocs having a nonuniform structure is estimated. A Darcy-Brinkman model is adopted to simulate the flow field inside a floc and a two-layer model used to describe the floc structure. The influences of the key parameters of the system under consideration, including the Reynolds number, the separation distance between two flocs, the relative magnitudes of the permeabilities of the inner and the outer layers of a floc, and the relative thickness of the inner and the outer layers of a floc, on the drag acting on two flocs are investigated.

\section{Theory}

We consider here two flocs moving along their centerline at a steady speed of $V$ through an unbound quiescent Newtonian fluid of viscosity $\mu$ and density $\rho$. For simplicity, we assume that a floc can be simulated by a two-layer structure. Referring to Fig. 1, let $d, r_{\mathrm{i}},\left(r_{\mathrm{o}}-r_{\mathrm{i}}\right)$, and $S$ be, respectively, the diameter of a floc, the radius of the inner layer, the thickness of the outer layer, and the center-to-center distance.

\subsection{Flow field in liquid phase}

Suppose that the steady-state flow field in the liquid phase can be described by the Navier-Stokes equation and the continuity equation

$\mathbf{u}_{\mathrm{f}} \cdot \nabla \mathbf{u}_{\mathrm{f}}=-\nabla P+\frac{2}{R e} \nabla^{2} \mathbf{u}_{\mathrm{f}}$,

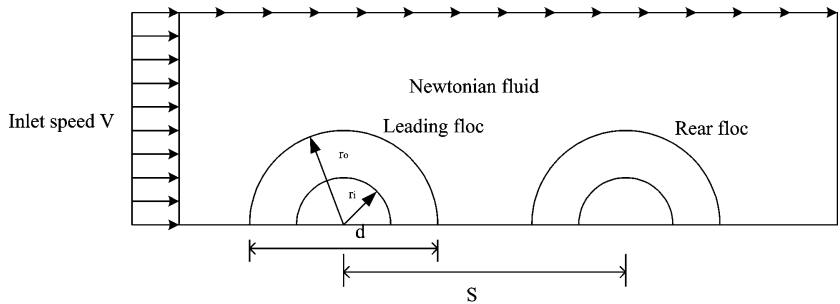

Fig. 1. Schematic representation of the problem considered where two identical, coaxial flocs are placed in an infinite Newtonian fluid. $r_{\mathrm{O}}$ and $r_{\mathrm{i}}$ are respectively the radii of the outer and the inner layers of a floc, and $V$ is the bulk velocity of the fluid.

$\nabla \cdot \mathbf{u}_{\mathrm{f}}=0$

where $R e=2 \rho r_{\mathrm{o}} V_{z} / \mu$ is the Reynolds number and $\rho, \mu$, and $V_{z}$ are respectively the density and the viscosity of fluid and the $z$-component of $\mathbf{V} . P=(p+\rho g Z) / \rho V_{z}^{2}$ is the scaled pressure, and $p, g$, and $Z$ are respectively the pressure, gravitational acceleration, and $z$-coordinate. $\nabla$ is the dimensionless gradient operator scaled by $1 / r_{\mathrm{o}}$, and $\mathbf{u}_{\mathrm{f}}$ is the dimensionless velocity scaled by $\mathbf{V}$.

\subsection{Flow field in inner and outer layers of a floc}

Suppose that the steady-state flow field inside a floc can be described by the Darcy-Brinkman model [2] and the continuity equation. That is,

$u_{j}+\frac{R e}{2 \beta_{j}^{2}} \nabla P=\nabla^{2} u_{j}, \quad j=\mathrm{o}, \mathrm{i}$,

$\nabla \cdot u_{j}=0, \quad j=\mathrm{o}, \mathrm{i}$,

where $\beta_{j}=r_{\mathrm{o}} / \sqrt{k_{j}}$ is the scaled floc radius, $k_{j}$ being the permeability of region $j, u_{j}$ is the scaled flow velocity in region $j$, scaled by $\mathbf{V}$, and $j$ is a region index, $j=0$ and $\mathrm{i}$ representing, respectively, the outer and the inner part of the floc.

\subsection{Boundary conditions}

We assume that both the fluid velocity and the shear stress are continuous on both the outer layer-liquid interface and the inner layer-outer layer interface. Also, the fluid far away from flocs is uninfluenced by their presence. Therefore, the boundary conditions associated with Eqs. (3)-(6) are

$\mathbf{u}_{\mathrm{f}}=\mathbf{u}_{\mathrm{o}} \quad$ and $\quad \mu_{\mathrm{f}} \nabla u_{\mathrm{f}}=\mu_{\mathrm{o}} \nabla u_{\mathrm{o}}, \quad r=r_{\mathrm{o}}$,

$\mathbf{u}_{\mathrm{o}}=\mathbf{u}_{\mathrm{i}} \quad$ and $\quad \mu_{\mathrm{o}} \nabla u_{\mathrm{o}}=\mu_{\mathrm{i}} \nabla u_{\mathrm{i}}, \quad r=r_{\mathrm{i}}$,

$u_{z}=1 \quad$ as $r \rightarrow \infty$,

where $u_{z}$ is the scaled $z$-component of the fluid velocity. The symmetric nature of the present problem also requires that

$\frac{\partial u_{\mathrm{f}}}{\partial r}=\frac{\partial u_{\mathrm{o}}}{\partial r}=\frac{\partial u_{\mathrm{i}}}{\partial r}=0, \quad r=0$.

For simplicity, we assume that $\mu_{\mathrm{f}}=\mu_{\mathrm{o}}=\mu_{\mathrm{i}}$. 
Table 1

Variation of the drag on two coaxial rigid spheres at various combinations of $S / d$ and Re calculated by the analytic result of Happel and Brenner [27] and by the present numerical method

\begin{tabular}{|c|c|c|c|c|c|}
\hline$S / d$ & $\begin{array}{l}\text { Analytic } \\
\text { result }(\mathrm{N})\end{array}$ & $\begin{array}{l}\text { Leading floc, } \\
\text { numerical (N) }\end{array}$ & $\begin{array}{l}\text { Percentage } \\
\text { deviation }(\%)\end{array}$ & $\begin{array}{l}\text { Rear floc, } \\
\text { numerical }(\mathrm{N})\end{array}$ & $\begin{array}{l}\text { Percentage } \\
\text { deviation }(\%)\end{array}$ \\
\hline \multicolumn{6}{|c|}{$R e=0.1$} \\
\hline 1.5 & $6.57867 \times 10^{-5}$ & $6.63629 \times 10^{-5}$ & -0.875844 & $6.68942 \times 10^{-5}$ & -1.6835301 \\
\hline 2 & $6.96812 \times 10^{-5}$ & $7.05898 \times 10^{-5}$ & -1.3039484 & $7.03209 \times 10^{-5}$ & -0.9181196 \\
\hline 3 & $7.53240 \times 10^{-5}$ & $7.73846 \times 10^{-5}$ & -2.7355871 & $7.64361 \times 10^{-5}$ & -1.4762946 \\
\hline 5 & $8.15366 \times 10^{-5}$ & $8.37071 \times 10^{-5}$ & -2.6619664 & $8.14296 \times 10^{-5}$ & 0.1312083 \\
\hline 11 & $8.86016 \times 10^{-5}$ & $8.97825 \times 10^{-5}$ & -1.3327904 & $8.72675 \times 10^{-5}$ & 1.5057354 \\
\hline \multicolumn{6}{|c|}{$\operatorname{Re}=0.01$} \\
\hline 1.5 & $6.57867 \times 10^{-6}$ & $6.55843 \times 10^{-6}$ & 0.3075983 & $6.74696 \times 10^{-6}$ & -2.5581599 \\
\hline 2 & $6.96812 \times 10^{-6}$ & $6.97742 \times 10^{-6}$ & -0.1334601 & $7.09281 \times 10^{-6}$ & -1.789431 \\
\hline 3 & $7.53240 \times 10^{-6}$ & $7.65238 \times 10^{-6}$ & -1.5927515 & $7.70815 \times 10^{-6}$ & -2.3332322 \\
\hline 5 & $8.15366 \times 10^{-6}$ & $8.29130 \times 10^{-6}$ & -1.6880849 & $8.20307 \times 10^{-6}$ & -0.605933 \\
\hline 11 & $8.86016 \times 10^{-6}$ & $8.93592 \times 10^{-6}$ & -0.8550565 & $8.75283 \times 10^{-6}$ & 1.2113728 \\
\hline
\end{tabular}

Note. Key: $r_{\mathrm{o}}=0.12 \mathrm{~cm}, r_{\mathrm{i}}=0.06 \mathrm{~cm}, \rho=1 \mathrm{~g} / \mathrm{cm}^{3}$, and $\mu=0.01$ poise.

\section{Results and discussion}

The governing equations and the associated boundary conditions were solved numerically by FIDAP 7.6, which is based on a finite element method. The applicability of this software is justified by comparing the results for the case of two rigid spheres with the analytic results of Happel and Brenner [27], which is exact as $R e \rightarrow 0$; both results are summarized in Table 1. Note that under the conditions of creeping flow, the drags on the leading particle should be the same as that on the rear particle. Table 1 reveals that these drags, while close to each other, are not exactly the same. This arises mainly from the fact that $R e$ has a small nonzero value and the precision limit of the software. The deviation is less than $3 \%$, suggesting that the performance of the software adopted is satisfactory. In general, the smaller the $R e$ the more accurate the analytic result for the leading floc is, and the reserve trend is observed for the rear floc. The former is expected, and the latter arises from the fact the behavior of the rear floc is inevitably influenced by the presence of the leading floc.

\subsection{Velocity field}

We define the volume-averaged permeability $\bar{k}$ and the volume-averaged radius $\bar{\beta}$ as

$$
\begin{aligned}
& \bar{k}=\sum_{j=1}^{2} V_{j} k_{j} / \sum_{j=1}^{2} V_{j}, \\
& \bar{\beta}=d_{\mathrm{p}} / 2 \sqrt{\bar{k}} .
\end{aligned}
$$

Fig. 2 shows the velocity field for two identical flocs at various $\left(k_{\mathrm{o}} / k_{\mathrm{i}}\right)$ for two levels of $R e$ for the case when $(S / d)$ is relatively small; that for the case when $(S / d)$ is relatively large is presented in Fig. 3. Here, because both $r_{\mathrm{o}}$ and $\bar{\beta}$ are fixed, so is the volume-averaged permeability of a floc. In Figs. $2 \mathrm{a}$ and $2 \mathrm{~d}, k_{\mathrm{o}} / k_{\mathrm{i}}=0.1$, the inner layer of a floc is more permeable than its outer layer; $k_{\mathrm{o}} / k_{\mathrm{i}}=1$ in Figs. $2 \mathrm{~b}$ and $2 \mathrm{e}$, where a floc has a uniform structure; $k_{\mathrm{o}} / k_{\mathrm{i}}=10 \mathrm{in}$ Figs. $2 \mathrm{c}$ and $2 \mathrm{f}$, where the inner layer of a floc is less permeable that its outer layer. As can be seen in Figs. 2a-2c, if $R e$ is small, the convective motion of fluid is inappreciable, and the flow field in front of a floc is symmetric to that on its back. As shown in Figs. 2d-2f, the flow field becomes asymmetric when $R e$ increases to 40 . However, due to the high permeability nature of a floc, boundary layer separation is not observed in the rear region of a floc. Fig. 2 also reveals that the flow field for the case when $k_{\mathrm{o}} / k_{\mathrm{i}}=0.1$ is different from that when $k_{\mathrm{o}} / k_{\mathrm{i}}=10$, implying that although the volume-averaged permeability of a floc remains the same, the flow field is still influenced by its structure. The qualitative behavior of the flow field in Fig. 3 is similar to that in Fig. 2. As can be seen in Figs. $3 \mathrm{a}-3 \mathrm{c}$, if $R e=0.1$, the interaction between the flow field of the leading floc and that of the rear floc becomes unimportant when $S / d=11$. However, this interaction is still appreciable when $R e=40$, as shown in Figs. 3d-3f, but is less significant than that for the case when $S / d=2$, as can be seen in Fig. 2 .

\subsection{Effect of separation distance between two flocs}

The influence of $(S / d)$ on the ratio $\left(C_{\mathrm{D}} \Omega_{\mathrm{r}} / C_{\mathrm{D}} \Omega_{1}\right)$ at various values of $\left(k_{\mathrm{o}} / k_{\mathrm{i}}\right)$ is illustrates in Fig. 4 for the case when $R e=40$ and $\bar{\beta}=2$. If $R e$ is sufficiently large, wakes are formed in the rear region of the leading floc and the drag on the rear floc is smaller than that when the leading floc is absent, as can be seen in Fig. 4. Also, the more nonuniform the floc structure, the more significant the influence of $(S / d)$ on the interaction between two flocs is. According to $\mathrm{Wu}$ and Lee [11], for uniformly structured flocs at $\beta=5$ and $R e=40$, the drags on both the leading and the rear flocs increase with the increase in $S / d$. However, because the former approaches a constant value but the latter does not, $\left(C_{\mathrm{D}} \Omega_{\mathrm{r}} / C_{\mathrm{D}} \Omega_{1}\right)$ increases with $(S / d)$, which is consistent with the result in Fig. 4. Furthermore, if $R e$ is suffi- 


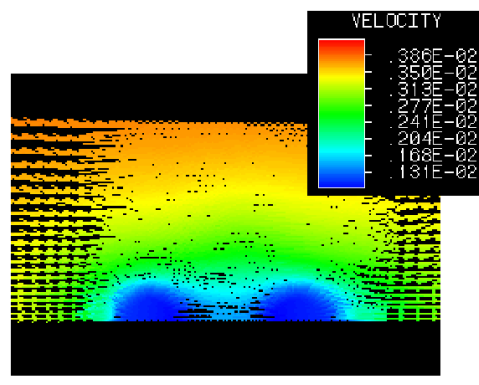

(a)

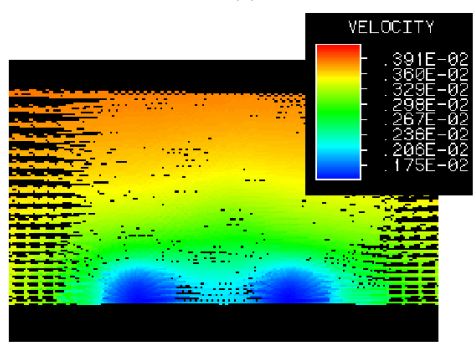

(b)

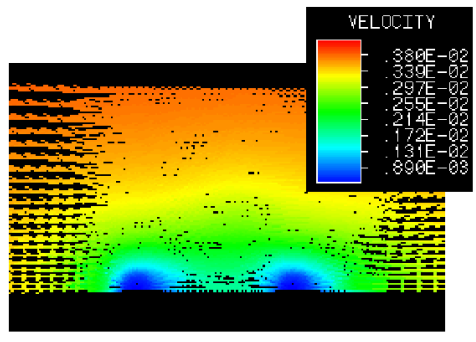

(c)

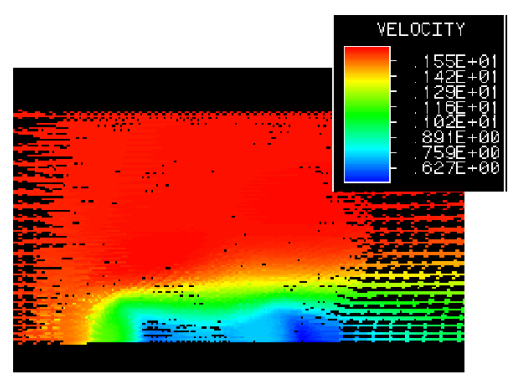

(d)

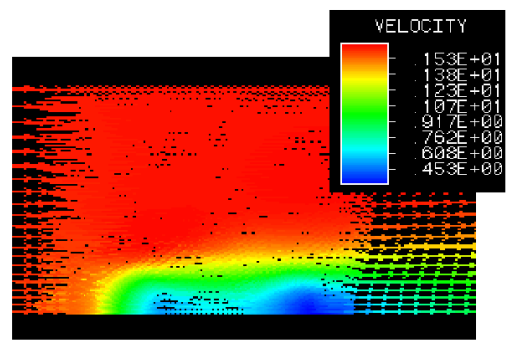

(e)

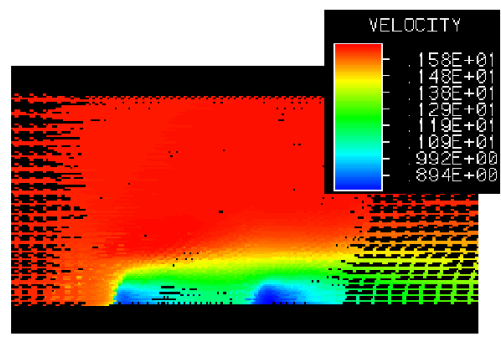

(f)

Fig. 2. Velocity field for two identical flocs at various $\left(k_{\mathrm{o}} / k_{\mathrm{i}}\right)$ for the case when $S / d=2$ and $\bar{\beta}=2 . \operatorname{Re}=0.1$ in (a), (b), (c), and $\operatorname{Re}=40$ in (d), (e), (f). (a) and (d), $k_{\mathrm{O}} / k_{\mathrm{i}}=0.1$; (b) and (e), $k_{\mathrm{O}} / k_{\mathrm{i}}=1$; (c) and (f), $k_{\mathrm{O}} / k_{\mathrm{i}}=10$. Key: $r_{\mathrm{O}}=0.12 \mathrm{~cm}, r_{\mathrm{i}}=0.06 \mathrm{~cm}, \rho=1 \mathrm{~g} / \mathrm{cm}^{3}$, and $\mu=0.01 \mathrm{poise}$.

ciently large, regardless of the value of $(S / d), C_{\mathrm{D}} \Omega_{\mathrm{r}}$ is always smaller than $C_{\mathrm{D}} \Omega_{1}$, and therefore, $\left(C_{\mathrm{D}} \Omega_{\mathrm{r}} / C_{\mathrm{D}} \Omega_{1}\right)<1$.

\subsection{Effect of Reynolds number}

The variation of the ratio $\left(C_{\mathrm{D}} \Omega_{\mathrm{r}} / C_{\mathrm{D}} \Omega_{1}\right)$ as a function of $R e$ at various combinations of $\left(k_{\mathrm{o}} / k_{\mathrm{i}}\right)$ and $(S / d)$ for a fixed $\bar{\beta}$ is illustrated in Fig. 5, and that at various $\left(k_{\mathrm{o}} / k_{\mathrm{i}}\right)$ when both $(S / d)$ and $\bar{\beta}$ are fixed is shown in Fig. 6. Fig. 5 suggests that if $R e$ is small, that is, the flow is close to creeping flow, the drags on the two flocs are about the same, as expected. However, as $R e$ increases, the influence of the flow field behind the leading floc on the rear floc becomes significant, and $\left(C_{\mathrm{D}} \Omega_{\mathrm{r}} / C_{\mathrm{D}} \Omega_{1}\right)$ starts to deviate from unity. Note that the more nonuniform the floc structure is, the more significant this effect. As can be seen in Fig. 5, for $\bar{\beta}=2,\left(C_{\mathrm{D}} \Omega_{\mathrm{r}} / C_{\mathrm{D}} \Omega_{1}\right)$ declines monotonically with the increase in $R e$, and approaches a constant when it is sufficiently large; the smaller the $(S / d)$, the smaller the $R e$ at which $\left(C_{\mathrm{D}} \Omega_{\mathrm{r}} / C_{\mathrm{D}} \Omega_{1}\right)$ starts to level off. In general, if $(S / d)$ is small, the influence of $R e$ on the rear floc is more important than that when it is large. It is interesting to observe in Fig. 6 that if $\bar{\beta}=1,\left(C_{\mathrm{D}} \Omega_{\mathrm{r}} / C_{\mathrm{D}} \Omega_{1}\right)$ has a local minimum as $R e$ varies, which does not appear for the case of two rigid spheres, and it is easier for this local minimum to appear when the inner layer of a floc is more permeable than its outer layer. The presence of the local minimum can be explained as follows. In general, when $R e$ is small, we have a creeping flow, and the drags on the two flocs are about the same, $\left(C_{\mathrm{D}} \Omega_{\mathrm{r}} / C_{\mathrm{D}} \Omega_{1}\right)$ is close to unity. As Re increases, the noncreeping flow field behind the leading floc leads to a smaller drag on the rear floc than that on the leading floc, $\left(C_{\mathrm{D}} \Omega_{\mathrm{r}} / C_{\mathrm{D}} \Omega_{1}\right)$ is smaller than unity, and the larger $R e$ the smaller $\left(C_{\mathrm{D}} \Omega_{\mathrm{r}} / C_{\mathrm{D}} \Omega_{1}\right)$. However, if the permeability of the floc is sufficiently high and $R e$ increases to a certain level, because it is easier for fluid to penetrate the leading floc, the influence of the flow field on the decline of the drag on the rear floc becomes less important and $\left(C_{\mathrm{D}} \Omega_{\mathrm{r}} / C_{\mathrm{D}} \Omega_{1}\right)$ becomes closer to 1 . Note that the local minimum does not occur if $k_{\mathrm{o}} / k_{\mathrm{i}}$ is sufficiently large (say, 10). This is because in this case the presence of the outer layer can be neglected and the inner layer is close to a rigid sphere. Fig. 6 also suggests that, if the outer layer of a floc is less permeable than its inner layer $\left(k_{\mathrm{o}} / k_{\mathrm{i}}<1\right)$, the larger the value of $k_{\mathrm{o}}$ the greater the ratio $\left(C_{\mathrm{D}} \Omega_{\mathrm{r}} / C_{\mathrm{D}} \Omega_{1}\right)$ is. On the other hand, if the outer layer of a floc is more permeable than its inner layer $\left(k_{\mathrm{o}} / k_{\mathrm{i}}>1\right)$, the larger the value of $k_{\mathrm{o}}$ the smaller the 


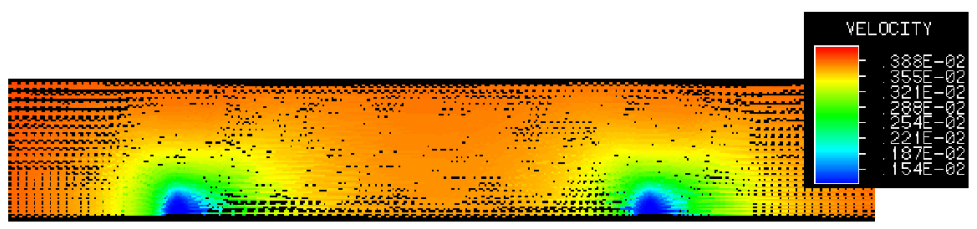

(a)

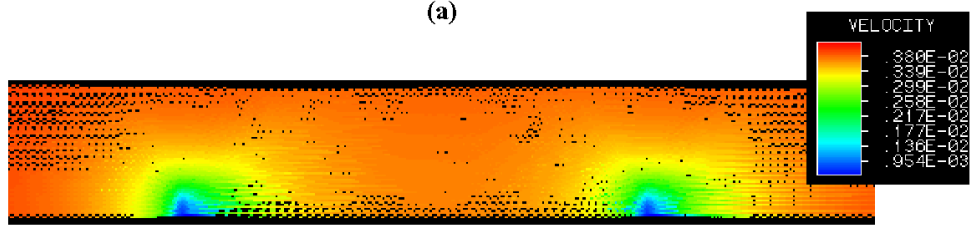

(b)

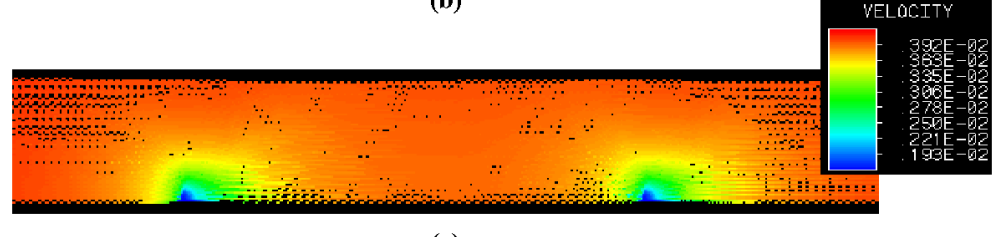

(c)

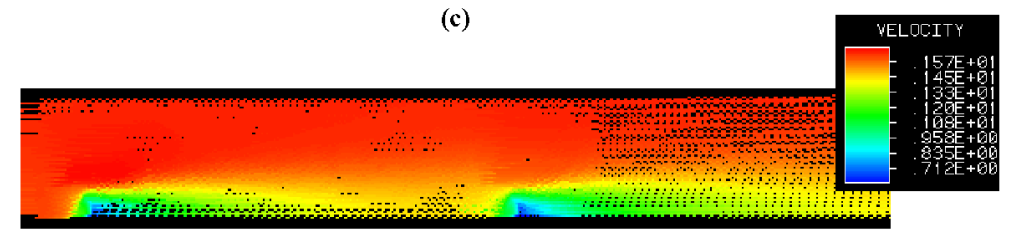

(d)

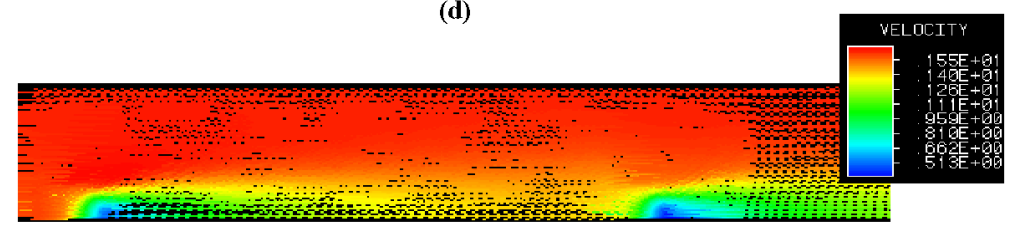

(e)

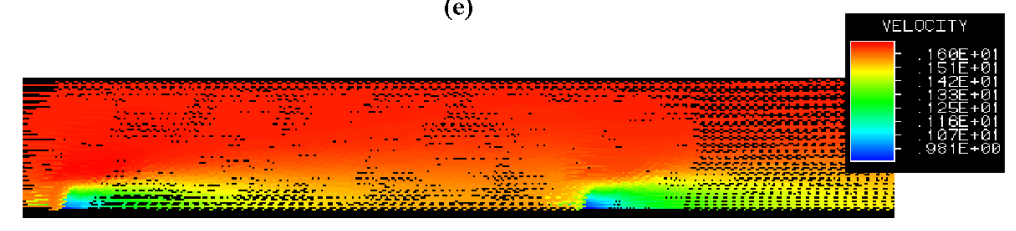

(f)

Fig. 3. Velocity field for two identical flocs at various $\left(k_{\mathrm{o}} / k_{\mathrm{i}}\right)$ for the case when $S / d=11$ and $\bar{\beta}=2 . R e=0.1$ in (a), (b), (c), and $R e=40$ in (d), (e), (f). (a) and (d), $k_{\mathrm{o}} / k_{\mathrm{i}}=0.1$; (b) and (e), $k_{\mathrm{o}} / k_{\mathrm{i}}=1$; (c) and (f), $k_{\mathrm{o}} / k_{\mathrm{i}}=10$. Key: same as in Fig. 2.

ratio $\left(C_{\mathrm{D}} \Omega_{\mathrm{r}} / C_{\mathrm{D}} \Omega_{1}\right)$ is. These observations reveal that the influences of the inner and the outer structures of a floc on $\left(C_{\mathrm{D}} \Omega_{\mathrm{r}} / C_{\mathrm{D}} \Omega_{1}\right)$ need to be considered simultaneously.

For creeping flow, the drag coefficient for a rigid sphere, $C_{\mathrm{D}}$, is described by Stokes' law,

$C_{\mathrm{D}}=\frac{24}{R e}$.

For a porous sphere, this expression can be modified as

$C_{\mathrm{D}} \Omega=\frac{A\left(\bar{\beta}, k_{\mathrm{o}} / k_{\mathrm{i}}, r_{\mathrm{o}} / R\right)}{\operatorname{Re}}$.

In this Stokes'-law-like relation $A$ is a function of $\bar{\beta},\left(k_{\mathrm{o}} / k_{\mathrm{i}}\right)$, and $\left(r_{\mathrm{o}} / R\right)$.

Figs. 7-9 illustrate the variations of $C_{\mathrm{D}} \Omega_{\mathrm{r}}$ and $C_{\mathrm{D}} \Omega_{1}$ as a function of $\operatorname{Re}$ for various combinations of $\left(k_{\mathrm{o}} / k_{\mathrm{i}}\right)$ and $\bar{\beta}$. These figures indicate that the smaller the $\bar{\beta}$ the closer a

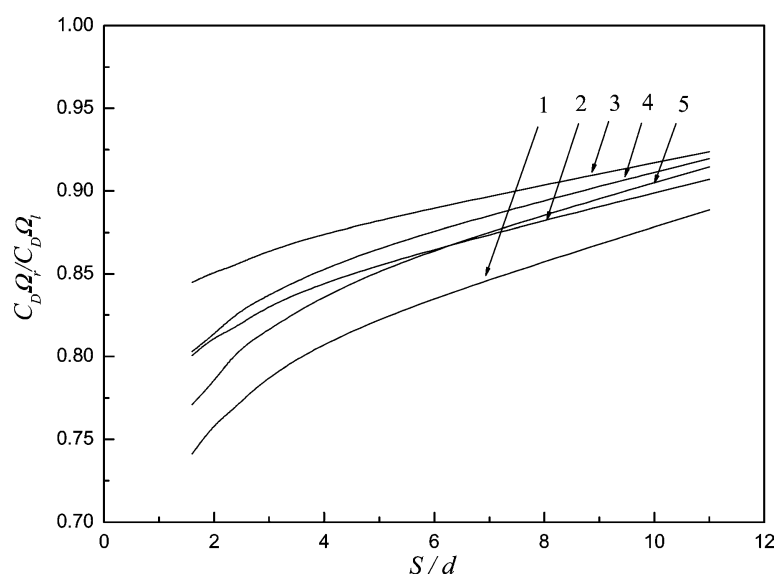

Fig. 4. Variation of $\left(C_{\mathrm{D}} \Omega_{\mathrm{r}} / C_{\mathrm{D}} \Omega_{1}\right)$ as a function of $(S / d)$ for various $\left(k_{\mathrm{o}} / k_{\mathrm{i}}\right)$ at $R e=40$ and $\bar{\beta}=2$. Curve $1, k_{\mathrm{o}} / k_{\mathrm{i}}=0.1 ; 2, k_{\mathrm{o}} / k_{\mathrm{i}}=0.2 ; 3$, $k_{\mathrm{o}} / k_{\mathrm{i}}=1 ; 4, k_{\mathrm{o}} / k_{\mathrm{i}}=5 ; 5, k_{\mathrm{o}} / k_{\mathrm{i}}=10$. Key: same as in Fig. 2 . 

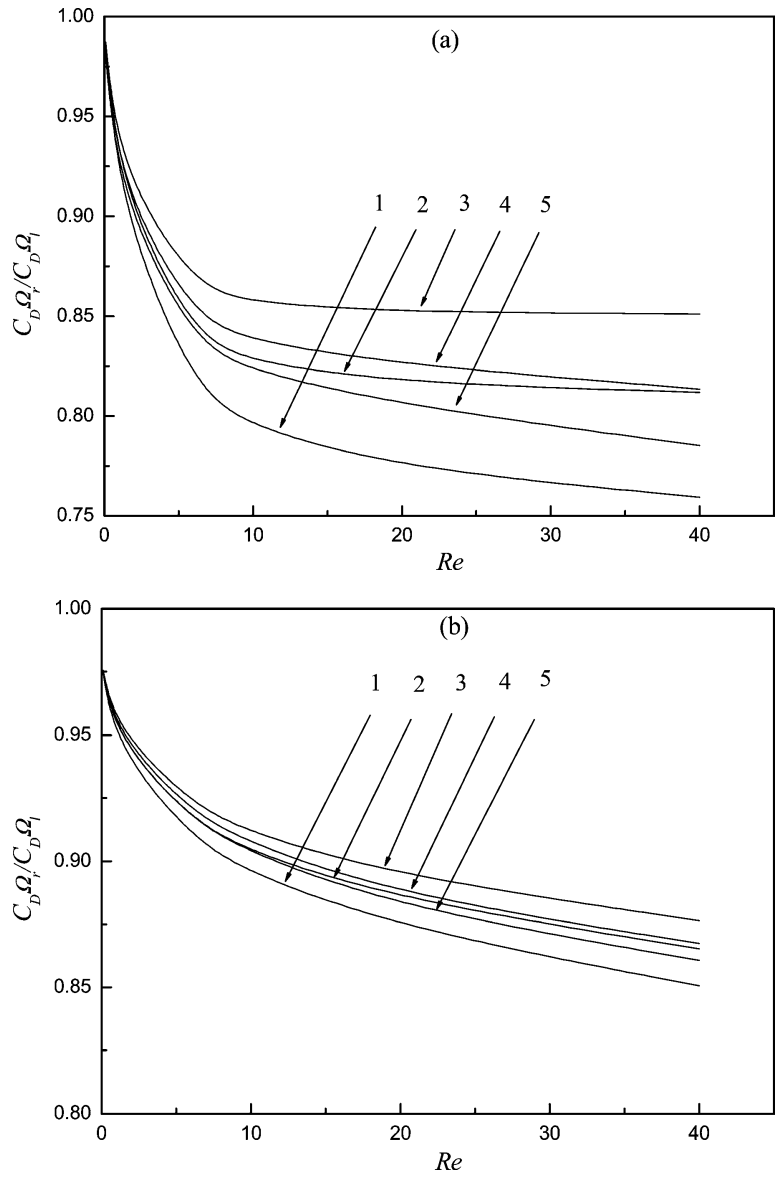

Fig. 5. Variation of $\left(C_{\mathrm{D}} \Omega_{\mathrm{r}} / C_{\mathrm{D}} \Omega_{1}\right)$ as a function of Re for various $\left(k_{\mathrm{o}} / k_{\mathrm{i}}\right)$ at different $(S / d)$ for the case when $\bar{\beta}=2$. Curve $1, k_{\mathrm{o}} / k_{\mathrm{i}}=0.1 ; 2$, $k_{\mathrm{o}} / k_{\mathrm{i}}=0.2 ; 3, k_{\mathrm{o}} / k_{\mathrm{i}}=1 ; 4, k_{\mathrm{o}} / k_{\mathrm{i}}=5 ; 5, k_{\mathrm{o}} / k_{\mathrm{i}}=10$. (a) $S / d=2$, (b) $S / d=11$. Key: same as in Fig. 2.

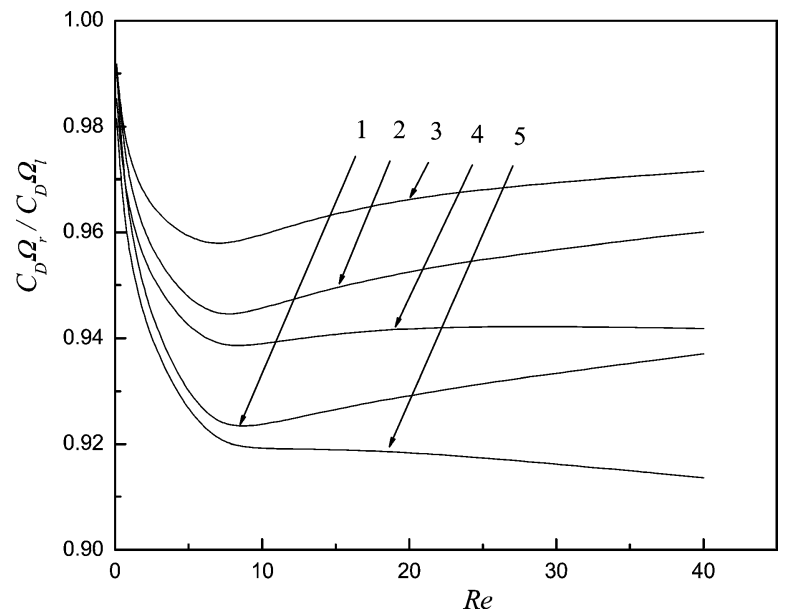

Fig. 6. Variation of $\left(C_{\mathrm{D}} \Omega_{\mathrm{r}} / C_{\mathrm{D}} \Omega_{1}\right)$ as a function of $R e$ for various $\left(k_{\mathrm{o}} / k_{\mathrm{i}}\right)$ for the case when $\bar{\beta}=1$ and $S / d=2$. Curve $1, k_{\mathrm{o}} / k_{\mathrm{i}}=0.1 ; 2, k_{\mathrm{o}} / k_{\mathrm{i}}=0.2$; $3, k_{\mathrm{o}} / k_{\mathrm{i}}=1 ; 4, k_{\mathrm{O}} / k_{\mathrm{i}}=5 ; 5, k_{\mathrm{o}} / k_{\mathrm{i}}=10$. Key: same as in Fig. 2.

$C_{\mathrm{D}} \Omega-R e$ curve to a Stokes'-law-like relation, which is expected because the greater the volume-averaged permeability of a floc the more uneasy for wakes to form behind it. Table 2 summarizes the deviations of $C_{\mathrm{D}} \Omega$ from a Stokes'-
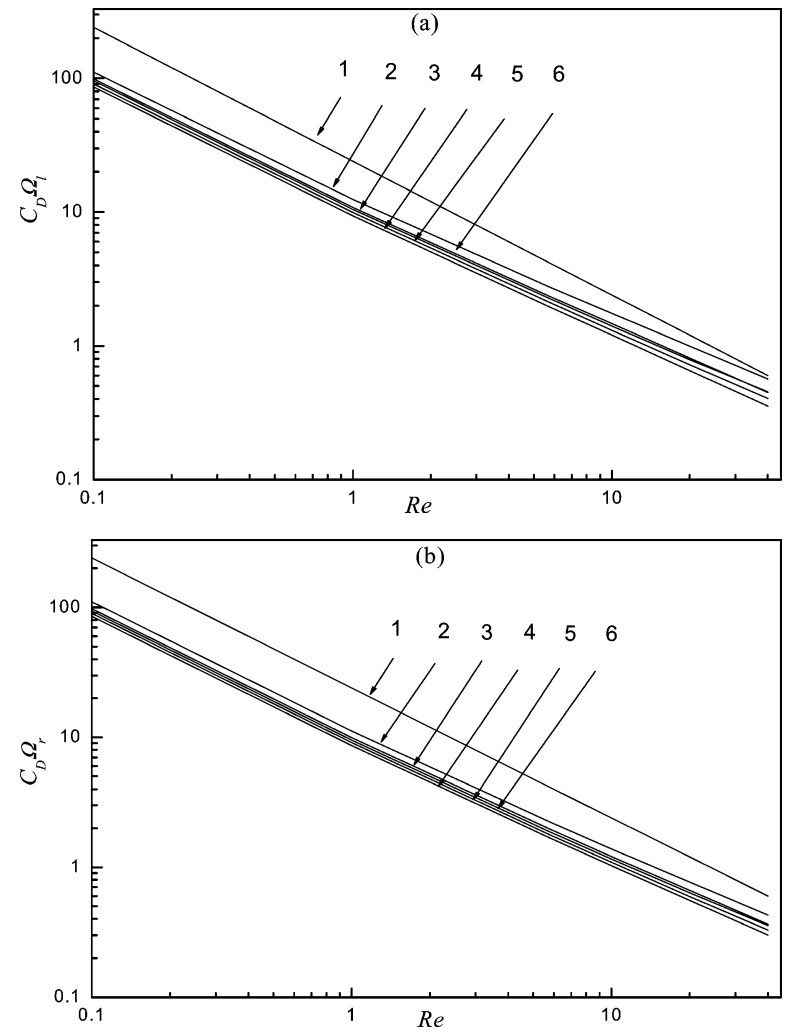

Fig. 7. Variation of $C_{\mathrm{D}} \Omega$ as a function of $R e$ for various $\left(k_{\mathrm{o}} / k_{\mathrm{i}}\right)$ for the case when $S / d=2$ and $\bar{\beta}=2$. Curve 1 , Stokes' law; $2, k_{\mathrm{o}} / k_{\mathrm{i}}=0.1 ; 3$, $k_{\mathrm{o}} / k_{\mathrm{i}}=0.2 ; 4, k_{\mathrm{o}} / k_{\mathrm{i}}=1 ; 5, k_{\mathrm{o}} / k_{\mathrm{i}}=5 ; 6, k_{\mathrm{o}} / k_{\mathrm{i}}=10$. (a) Leading floc, (b) rear floc. Key: same as in Fig. 2.

law-like relation at $R e=40$ for various $\bar{\beta}$. For a rigid sphere, Stokes' law is applicable for $R e$ in the range of creeping flow. In the present case, the porous nature of a floc implies that a Stokes'-law-like relation can be followed for $R e$ larger than that for the case of rigid sphere. As can be seen in Table 2, the deviation of $C_{\mathrm{D}} \Omega$ at $R e=40$ increases with the increase in $\bar{\beta}$. No general rule is observed between the relative magnitudes of the deviations of $C_{\mathrm{D}} \Omega$ for the leading and the rear flocs. Judging from the degree of deviation of a curve from a straight line in Figs. 7-9, the larger the $\bar{\beta}$ and the more inhomogeneous the structure of a floc is, the more significant the presence of convective flow.

\subsection{Effect of floc structure}

Fig. 10 shows the variation of $\left(C_{\mathrm{D}} \Omega_{\mathrm{r}} / C_{\mathrm{D}} \Omega_{1}\right)$ as a function of $\left(k_{\mathrm{o}} / k_{\mathrm{i}}, R e\right)$ at two levels of $(S / d)$. According to this figure, regardless of the separation distance between two flocs, the more nonuniform the structures of flocs the smaller is $\left(C_{\mathrm{D}} \Omega_{\mathrm{r}} / C_{\mathrm{D}} \Omega_{1}\right)$. Fig. 10 also shows that the smaller the $(S / d)$ and/or the larger the $R e$, the more important the influence of the nonuniform structure of floc on $\left(C_{\mathrm{D}} \Omega_{\mathrm{r}} / C_{\mathrm{D}} \Omega_{1}\right)$. Fig. 11 illustrates the variation of $\left(C_{\mathrm{D}} \Omega_{\mathrm{r}} / C_{\mathrm{D}} \Omega_{1}\right)$ as a function of $\left(r_{\mathrm{i}} / r_{\mathrm{o}}\right)$ for various $\left(k_{\mathrm{o}} / k_{\mathrm{i}}\right)$. According to Eq. (11), if $\left(k_{\mathrm{o}} / k_{\mathrm{i}}\right)<1$ and the volume-averaged permeability of floc remains fixed, an increase in $\left(r_{\mathrm{i}} / r_{\mathrm{o}}\right)$ implies that the 

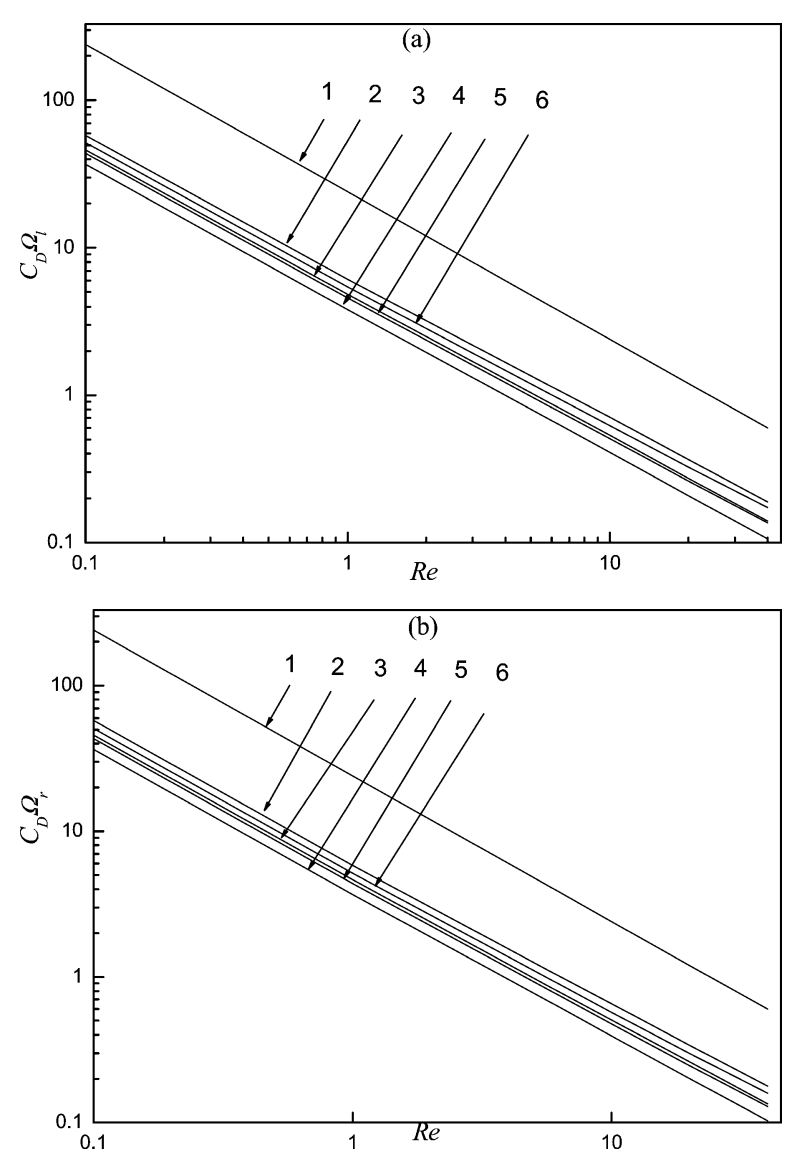

Fig. 8. Variation of $C_{\mathrm{D}} \Omega$ as a function of $R e$ at various $\left(k_{\mathrm{o}} / k_{\mathrm{i}}\right)$ for the case of Fig. 7, except that $\bar{\beta}=1$.

permeability of the outer layer of a floc decreases, which leads to a decrease in $\left(C_{\mathrm{D}} \Omega_{\mathrm{r}} / C_{\mathrm{D}} \Omega_{1}\right)$. On the other hand, if $\left(k_{\mathrm{o}} / k_{\mathrm{i}}\right)>1$, an increase in $\left(r_{\mathrm{i}} / r_{\mathrm{o}}\right)$ implies that the permeability of the outer layer of a floc increases, which leads to an increase in $\left(C_{\mathrm{D}} \Omega_{\mathrm{r}} / C_{\mathrm{D}} \Omega_{1}\right)$. In general, the influence of $\left(r_{\mathrm{i}} / r_{\mathrm{o}}\right)$ on $\left(C_{\mathrm{D}} \Omega_{\mathrm{r}} / C_{\mathrm{D}} \Omega_{1}\right)$ is more significant when $k_{\mathrm{o}}<k_{\mathrm{i}}$; that is, a floc has a more permeable inner layer.

\subsection{Effect of mean floc permeability}

The influence of $\left(k_{\mathrm{o}} / k_{\mathrm{i}}\right)$ on the variation of $\left(C_{\mathrm{D}} \Omega_{\mathrm{r}} /\right.$ $\left.C_{\mathrm{D}} \Omega_{1}\right)$ as a function of $\bar{\beta}$ is shown in Fig. 12. This figure indicates that the larger the value of $\bar{\beta}$ (the smaller the volumeaveraged permeability of a floc) the greater the difference between the drags on two flocs, and the more nonuniform the structure of a floc the more appreciable this effect is. Fig. 12 also suggests that the less permeable the outer layer of a floc (curves 2 and 3 ) the more significant the influence of $\bar{\beta}$ on the ratio $\left(C_{\mathrm{D}} \Omega_{\mathrm{r}} / C_{\mathrm{D}} \Omega_{1}\right)$.

In summary, the drag on two coaxial nonuniformly structured flocs in a uniform flow field is estimated by solving the governing equations for the flow field coupled with a two-layer model for floc structure for small to medium large Reynolds numbers. Based on the results of numerical simulation, we conclude the followings:
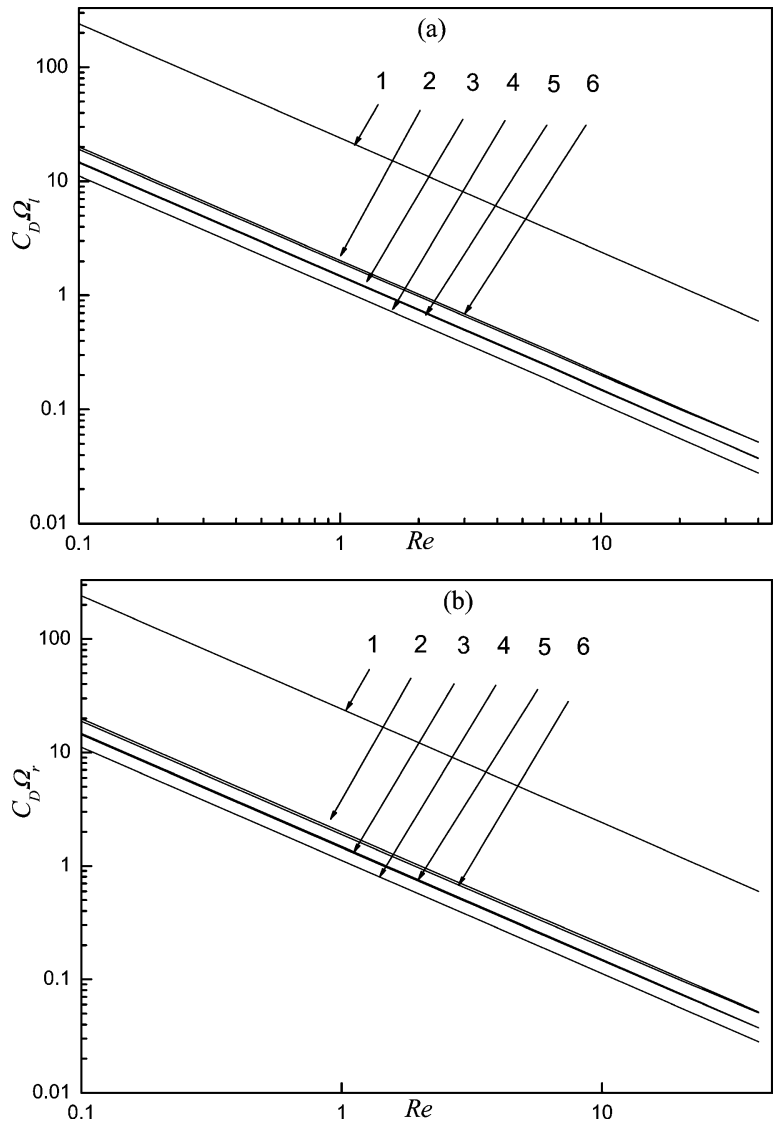

Fig. 9. Variation of $C_{\mathrm{D}} \Omega$ as a function of $R e$ at various $\left(k_{\mathrm{o}} / k_{\mathrm{i}}\right)$ for the case of Fig. 7, except that $\bar{\beta}=0.5$.

(a) At a low Reynolds number, the convective flow of fluid is unimportant and the flow field near the leading region of a floc is symmetric to that in its read region. In this case, the drag on the leading floc is the about the same as that on the rear floc. As Reynolds number is raised to 40 , the convective flow becomes appreciable, and the flow field near the leading region of a floc is asymmetric to that in its read region. Wakes are observed in the rear region of a floc, which has the effect of reducing the drag on the rear floc. This phenomenon is pronounced as the separation distance between two flocs declines. However, due the high permeability of a floc, boundary separation is inappreciable.

(b) If the permeability of floc is high, a drag coefficientReynolds number curve tends to follow a Stokes'-lawlike relation. A positive deviation from that relation is observed when the permeability of floc is low, and the more inhomogeneous the floc structure the more appreciable the deviation is.

(c) For a fixed volume-averaged permeability, the more inhomogeneous the floc structure, the more important the influence of Reynolds number on the ratio (drag on leading floc/drag on rear floc), especially when the outer layer of a floc is less permeable than its inner layer.

(d) The less permeable a floc is, the more the difference between the drags acting on two flocs, and this phe- 
Table 2

Percentage deviation from a Stokes'-law-like relation at $R e=40$ for various $\bar{\beta}$ for the case when $S / d=2$

\begin{tabular}{lccc}
\hline $\bar{\beta}$ & $k_{\mathrm{o}} / k_{\mathrm{i}}$ & \multicolumn{2}{c}{ Percentage deviation $(\%)$} \\
\cline { 3 - 4 } & & Leading floc & Rear floc \\
\hline 0.5 & 1 & -0.015 & 0.031 \\
0.5 & 0.1 & 0.165 & 0.205 \\
0.5 & 0.2 & 0.021 & 0.072 \\
0.5 & 10 & 0.396 & 0.370 \\
0.5 & 5 & 0.141 & 0.164 \\
1 & 1 & 1.399 & 1.181 \\
1 & 0.1 & 4.437 & 3.377 \\
1 & 0.2 & 2.427 & 1.964 \\
1 & 10 & 4.383 & 3.125 \\
1 & 5 & 2.665 & 2.035 \\
2 & 1 & 13.831 & 8.846 \\
2 & 0.1 & 28.686 & 15.425 \\
2 & 0.2 & 20.065 & 11.963 \\
2 & 10 & 21.333 & 12.070 \\
2 & 5 & 17.673 & 10.524 \\
\hline
\end{tabular}

Note. Key: same as in Fig. 2.

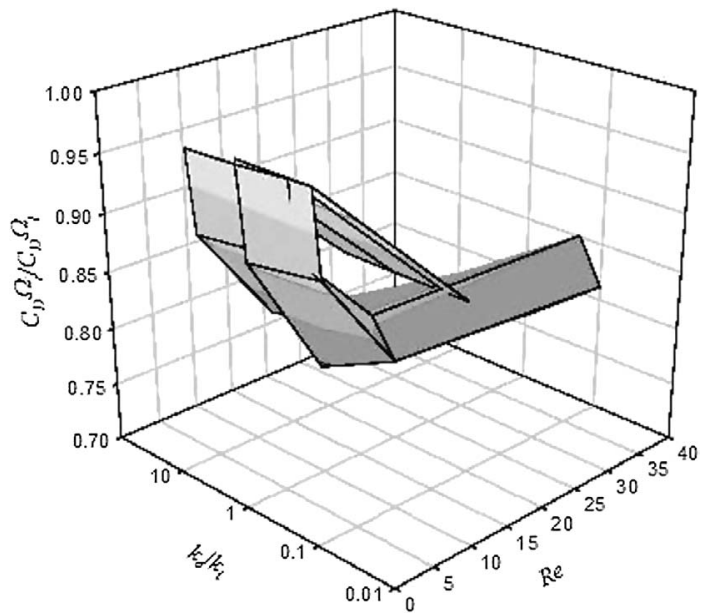

(a)

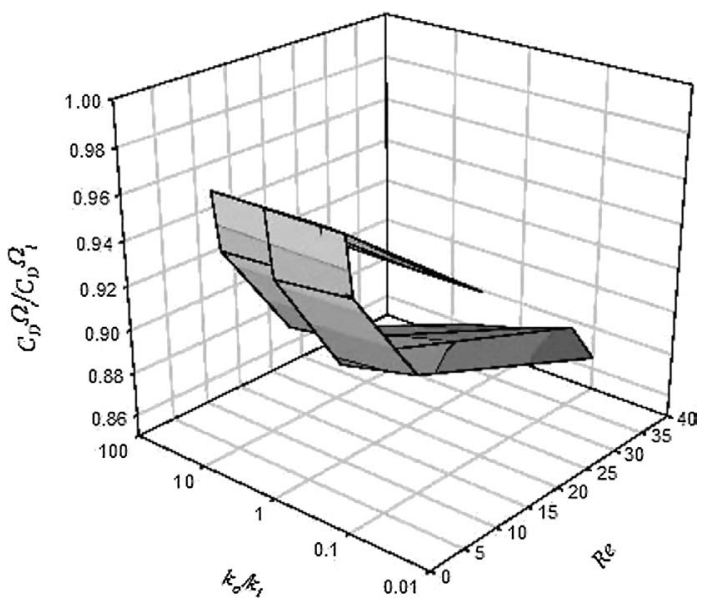

(b)

Fig. 10. Variation of $\left(C_{\mathrm{D}} \Omega_{\mathrm{r}} / C_{\mathrm{D}} \Omega_{1}\right)$ as a function of $\left(k_{\mathrm{o}} / k_{\mathrm{i}}, R e\right)$ at two levels of $(S / d)$ for the case when $\bar{\beta}=2$. (a) $S / d=2$, (b) $S / d=11$. Key: same as in Fig. 2.

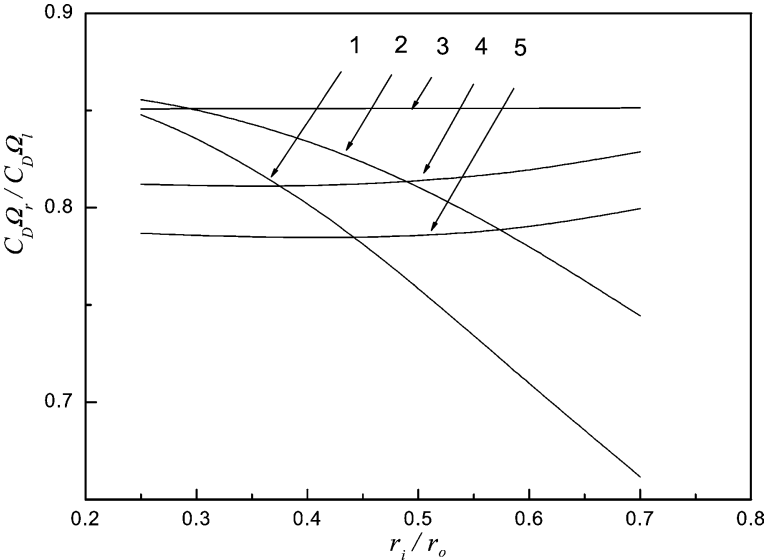

Fig. 11. Variation of $\left(C_{\mathrm{D}} \Omega_{\mathrm{r}} / C_{\mathrm{D}} \Omega_{1}\right)$ as a function of $\left(r_{\mathrm{i}} / r_{\mathrm{o}}\right)$ at various $\left(k_{\mathrm{o}} / k_{\mathrm{i}}\right)$ for the case when $S / d=2, \bar{\beta}=2$, and $R e=40$. Curve 1 , $k_{\mathrm{o}} / k_{\mathrm{i}}=0.1 ; 2, k_{\mathrm{o}} / k_{\mathrm{i}}=0.2 ; 3, k_{\mathrm{o}} / k_{\mathrm{i}}=1 ; 4, k_{\mathrm{o}} / k_{\mathrm{i}}=5 ; 5, k_{\mathrm{o}} / k_{\mathrm{i}}=10$. Key: same as in Fig. 2.

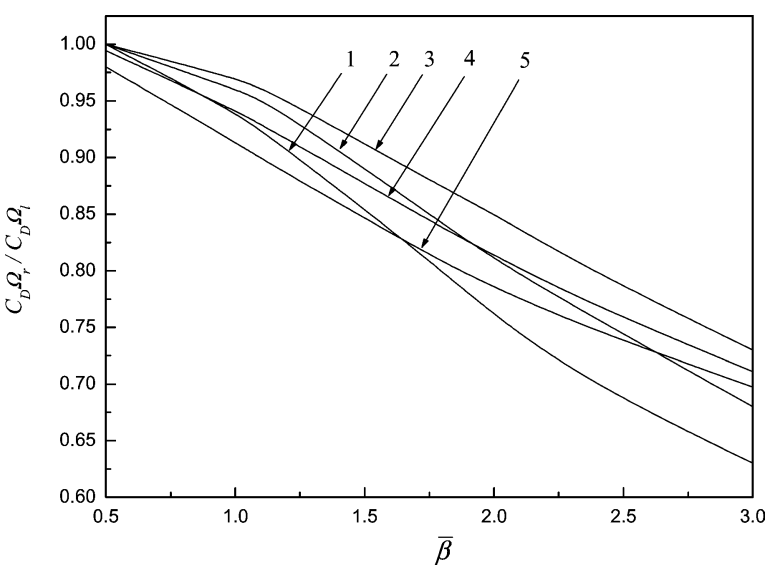

Fig. 12. Variation of $\left(C_{\mathrm{D}} \Omega_{\mathrm{r}} / C_{\mathrm{D}} \Omega_{1}\right)$ as a function of $\bar{\beta}$ at various $\left(k_{\mathrm{o}} / k_{\mathrm{i}}\right)$ for the case when $S / d=2$ and $R e=40$. Curve $1, k_{\mathrm{o}} / k_{\mathrm{i}}=0.1 ; 2, k_{\mathrm{o}} / k_{\mathrm{i}}=0.2$; $3, k_{\mathrm{o}} / k_{\mathrm{i}}=1 ; 4, k_{\mathrm{o}} / k_{\mathrm{i}}=5 ; 5, k_{\mathrm{o}} / k_{\mathrm{i}}=10$. Key: same as in Fig. 2 .

nomenon is more appreciable when floc is more nonuniformly structured, especially when the outer layer of a floc is less permeable than its inner layer.

(e) For a fixed volume-averaged permeability, the behavior of the ratio (drag on rear floc/drag on leading floc) as the ratio (radius of inner layer/radius of outer layer) varies for the case when the outer layer of a floc is less permeable than its inner layer is totally different from that when the outer layer is more permeable. In general, the influence of the ratio (radius of inner layer/radius of outer layer) on the ratio (drag on rear floc/drag on leading floc) is more important when the inner layer of a floc is more permeable than its outer layer.

\section{References}

[1] D.J. Lee, G.W. Chen, Y.C. Liao, C.C. Hsieh, Water Res. 30 (1996) 541. 
[2] G. Neale, N. Epstein, W. Nader, Chem. Eng. Sci. 28 (1973) 1865.

[3] R. Clift, J.R. Grace, M.E. Weber, Bubbles, Drops and Particles, Academic Press, New York, 1978.

[4] N. Tambo, Y. Watanabe, Water Res. 13 (1979) 409.

[5] J. Namer, J. Ganczarczyk, Water Res. 27 (1993) 1285.

[6] K. Matsumoto, A. Suganuma, Chem. Eng. Sci. 32 (1977) 445.

[7] C.P. Chu, D.J. Lee, Bull. College Eng. NTU 82 (2001) 49.

[8] R.M. Wu, D.J. Lee, Chem. Eng. Sci. 53 (1998) 3571.

[9] R.M. Wu, D.J. Lee, Chem. Eng. Sci. 54 (1999) 5717.

[10] R.M. Wu, D.J. Lee, Water Res. 35 (2001) 3226.

[11] R.M. Wu, D.J. Lee, Chem. Eng. Sci. 59 (2003) 943.

[12] F. Jorand, F. Zartarian, F. Thomas, J.C. Block, J.Y. Bottero, G. Villemin, V. Urbain, J. Manem, Water Res. 29 (1995) 1639.

[13] D.H. Li, J. Ganczarczyk, Biotechnol. Bioeng. 35 (1990) 57.

[14] D.H. Li, J. Ganczarczyk, Water Res. 22 (1988) 789.

[15] J.P. Hsu, Y.H. Hsieh, Chem. Eng. Sci. 57 (2002) 2627.
[16] J.P. Hsu, Y.H. Hsieh, J. Colloid Interface Sci. 259 (2003) 301.

[17] J.P. Hsu, Y.H. Hsieh, J. Colloid Interface Sci. 264 (2003) 517.

[18] J.P. Hsu, Y.H. Hsieh, J. Colloid Interface Sci. 275 (2004) 309.

[19] M.C. Li, M.S. thesis, National Taiwan University, Taipei, Taiwan, 2004.

[20] M. Stimson, G.B. Jeffery, Proc. R. Soc. London Ser. A 111 (1926) 110.

[21] P.N. Rowe, G.A. Henwood, Inst. Chem. Eng. 39 (1961) 43.

[22] K.C. Lee, Aerospace Q. 30 (1979) 371.

[23] Y. Tsuji, Y. Morikawa, K. Terashima, Int. J. Multiphase Flow 8 (1982) 71.

[24] C. Zhu, S.C. Liang, L.S. Fan, Int. J. Multiphase Flow 20 (1994) 117.

[25] S.C. Liang, T. Hong, L.S. Fan, Int. J. Multiphase Flow 22 (1996) 285.

[26] R.C. Chen, J.L. Wu, Chem. Eng. Sci. 55 (2000) 1143.

[27] J. Happel, H. Brenner, Low Reynolds Number Hydrodynamics, Academic Press, New York, 1983. 